\title{
La operacionalización del concepto de territorio en el INTA: los proyectos regionales con enfoque territorial
}

\section{Operationalization of the concept of territory at INTA: regional projects with a territorial approach}

Pablo SÁnchez Macchioli*

\begin{abstract}
The objective of this qualitative research was analyzing the incorporation of territorialization ideas by INTA, the way they were operationalized through a program denominated PRET and what institutional debates arose during the implementation of this policy. The main contribution of this work consisted in explaining in which way INTA made use of the territorial approach to adjust its governance to a political and social context which required a bigger interaction of its intervention policies in terms of the territories demands.
\end{abstract}

Keywords: territory, public policy, management.

\section{Resumen}

Esta investigación cualitativa tiene como objetivo analizar la incorporación de las ideas de territorialización en el Instituto Nacional de Tecnología Agropecuaria (INTA). Plantea cómo estas ideas fueron operacionalizadas a través de un instrumento programático que se denominó PRET y cuáles fueron los debates internos que surgieron en el INTA al momento de su creación. El principal aporte de este trabajo consistió en explicar de qué manera el enfoque territorial fue utilizado por el INTA para adaptar su gobernanza a un contexto político y social que demandaba una articulación más profunda de sus políticas de intervención con las demandas surgidas en los territorios.

Palabras clave: territorio, política pública, gestión.

*Instituto de Estudios sobre la Ciencia y la Tecnología de la Universidad Nacional de Quilmes, correo-e: psanchezmacchioli@uvq.edu.ar 


\section{Introducción}

El Instituto Nacional de Tecnología Agropecuaria (INTA) fue creado en 1956 con la finalidad de abordar problemáticas relacionadas con la investigación y la extensión agropecuaria, así como para contribuir a mejorar la tecnificación y eficiencia productiva del agro argentino ${ }^{1}$. La fundación de esta institución se suscitó en un momento histórico en el cual se crearon un conjunto de instituciones científicas y tecnológicas en la región, promovidas en buena parte por la Comisión Económica para América Latina y el Caribe (CEPAL), destinadas a la promoción del desarrollo social y económico. De esta manera, la fundación del INTA se erigió en un contexto más amplio, en el cual se fundaron centros en el país como la Comisión Nacional de Energía Atómica (CNEA), el Consejo Nacional de Investigaciones Científicas y Técnicas (CONICET) y la Comisión Nacional de Investigaciones Espaciales (CNIE, luego CONAE), entre otros.

Desde sus inicios, el INTA se constituyó como un organismo de una gran centralidad en el sistema científico y tecnológico argentino. El fuerte perfil agroexportador del país, que se mantuvo sin grandes modificaciones desde la conformación del estado argentino, llevó al INTA a ocupar un rol estratégico en las políticas públicas en relación con sectores como el agrícola-ganadero. Según Albornoz (2015) el INTA se convirtió, desde su fundación, en uno de los casos de mayor éxito, pues vincula varios sectores de la población: el estado, el sector académico y productivo. Esto se debe a que su estructura organizacional relaciona sistemáticamente la investigación con la extensión; sumado a la dispersión federal del instituto y a una gobernanza institucional que conjuga el sector público con el privado, lo cual le permitió optimizar su capacidad de interacción con el medio productivo y social.

La creación de la institución, como menciona Gárgano (2013), se enmarcó en una preocupación de la dictadura militar que tomó el poder en 1955 por la situación económica del país. Fue entonces cuando comenzó la aplicación de lo que se denominó Plan Prebisch, que tuvo como finalidad dar cuenta de algunos problemas estructurales de la economía argentina. El sector agrícola-ganadero, que presentaba un fuerte estancamiento productivo, se convirtió entonces en objeto de políticas públicas destinadas a intentar revertir esa tendencia; por lo que el INTA fue una de esas respuestas estatales. Como señala Scheinkerman de Obschatko (1988) la

${ }^{1}$ El INTA fue creado por el Decreto/Ley 21680/1956 del Poder Ejecutivo Nacional el 4 de diciembre de 1956. El Decreto de Ley fue ratificado por el Honorable Congreso de la Nación, a través de la Ley 14.467 de septiembre de 1958. Luego, una ley posterior, No 15.429, de agosto 1958, introdujo algunos ajustes. En 1961 el Consejo Directivo del INTA aprobó el texto definitivo. Texto de la ley disponible en: https://www.argentina.gob.ar/normativa/nacional/decreto_ley-21680-1956$77763 /$ texto 
instauración del INTA significó un cambio profundo en la conducción de la política tecnológica del sector agropecuario: por primera vez se creó una institución en el país orientada a la generación y difusión de tecnología agropecuaria.

Para el cumplimiento de sus funciones institucionales, el INTA implementó una serie de estrategias e instrumentos que han evolucionado a lo largo del tiempo y que pueden correlacionarse con los distintos procesos políticos y sociales que atravesó el país a lo largo de su historia. En sus primeras dos décadas de existencia, el INTA introdujo un enfoque que se enmarca en un paradigma "técnico-educativo", con una fuerte articulación entre la investigación y la extensión; a partir de la última dictadura cívicomilitar de 1976, el enfoque tuvo un mayor énfasis en la eficiencia y productividad, impulsando a los pequeños productores y a la agricultura familiar para priorizar la agricultura exportadora de gran escala; y por último, desde los primeros años del siglo XXI, se puede advertir que la institución se encontraba en un proceso interno de redefinición, en el cual el componente territorial ha comenzado a tener un peso muy importante en el discurso institucional y en las prácticas de investigación y extensión (Alemany, 2003: 144). El objetivo de este trabajo, por lo tanto, consiste en comprender de qué manera este componente territorial se discutió y se operacionalizó en el INTA, tomando a los proyectos regionales con enfoque territorial como estudio de caso, dado que es a partir de allí que el enfoque territorial se convierte expresamente en un criterio de orientación de las políticas internas de gestión de la investigación y de la extensión.

Con el objetivo realizar este estudio se llevó a cabo un análisis de una variedad de documentos institucionales publicados por el INTA, en particular de dos planes estratégicos institucionales, los cuales, a su vez, incluyen diversos documentos de trabajo llevados adelante por el Programa Nacional para el Desarrollo de los Territorios y otros programas nacionales de INTA. También se realizaron 26 entrevistas presenciales a investigadores, extensionistas, directores de grupos de investigación y extensión y directores de programas nacionales pertenecientes a INTA, las cuales tuvieron por objetivo conocer de primera mano las percepciones que tenían los distintos actores involucrados acerca del surgimiento e implementación de los PRET y en qué medida este instrumento surgió como una respuesta a discusiones históricas del INTA relacionadas a las preocupaciones sobre la eficacia de las intervenciones en los territorios.

El trabajo de investigación llevó a cabo su recolección de datos a través de dos técnicas principales: en primer lugar, el análisis de bibliografía sobre geografía rural y desarrollo territorial; así como el análisis de la documentación institucional del INTA y de páginas web institucionales. 
En segundo lugar, a partir de la realización de entrevistas semiestructuradas con agentes de investigación y extensión agropecuaria, así como gestores de la investigación dentro del INTA; también a participantes de PRET que no estaban incluidos en el grupo anterior e investigadores externos.

\section{Sobre el concepto de territorio}

El concepto de territorio ha propiciado innumerables reflexiones, especialmente desde la conformación del campo disciplinar de la geografía a finales del siglo XIX. Ratzel, geógrafo alemán, comenzó a conceptualizar el territorio en referencia al estado; su pensamiento positivista y darwiniano relacionaba el poder de una nación con su dimensión física y extensión territorial, en el contexto de la lucha europea por la supremacía económica y política, a la par del desarrollo del colonialismo a escala mundial (PaulsenBilbao, 2016: 67).

Luego, a mediados del siglo XX, este concepto perdió importancia y a la par la noción de región empezó a adquirir relevancia impulsada por la escuela de geografía francesa; allí comienza a cobrar mucha importancia la corriente que se denomina "posibilismo", que ponía el acento en la regionalización. En palabras de Ramírez (2017: 119):

\footnotetext{
Una vez terminada la Segunda Guerra Mundial, cuando el mundo precisaba de condiciones que le permitieran salir de la crisis de la posguerra, se requería de una unidad territorial que permitiera organizar la intervención del estado con fines de evolución y progreso. Para ello, la categoría de región dio una posibilidad importante, al integrarse a la de desarrollo, originando que se hablara de desarrolo regional, como la necesidad de generar regiones que permitieran promover la industrialización y, por lo tanto, la modernidad.
}

Esta visión regional se logró imponer y se convirtió en el paradigma que predominó en los estudios sociales del espacio hasta aproximadamente la década de 1970. A partir de ese momento se comenzó a recuperar la noción de territorio, de acuerdo con lo que Harvey (1990: 9) plantea como una "metamorfosis en las prácticas culturales y económico-políticas... ligada al surgimiento de nuevas formas dominantes de experimentar el espacio y el tiempo".

Esta modificación en las formas en las cuales se ejerce el poder y la dominación, a partir de los años 70, tiene un correlato espacial y temporal preciso, e hizo necesario tomar a los territorios y a los actores locales como una referencia ineludible en los análisis de los procesos de índole socioespacial. Según Manzanal (2008) el concepto de territorio comienza a tornarse recurrente, irrumpiendo muy fuertemente en los discursos insti- 
tucionales. La evolución en la conceptualización de territorio fue dejando de lado la impronta positivista, y fue incorporando dimensiones que se asocian a conceptos como poder, desarrollo y participación social. En este sentido, y de acuerdo con lo que plantea Lopes de Souza (1995: 81), "un territorio es un campo de fuerzas, una tela, una red de relaciones sociales, que a la par de su complejidad interna, define al mismo tiempo, un límite, una alteridad: la diferencia entre 'nosotros' (un grupo, los miembros de una comunidad) y los 'otros"'.

Estas nociones de territorio, al incorporar el problema del poder, se corresponden con las preocupaciones crecientes de los estados (a partir de la década de 1980) por la gestión de los fenómenos socioespaciales en una dimensión local. En relación con esto, algunos autores como Correia de Andrade (2002) ven al territorio como una conceptualización relacionada con la idea de dominio o gestión dentro de un espacio determinado; está ligado a la idea de poder público, estatal o privado en todas las escalas. Es aquí cuando se puede realizar un cruce entre las nociones de territorio y el concepto de desarrollo, el cual, necesariamente, se convierte en un proceso de espacialización diferenciada. Pensar al territorio como una red de relaciones nos permite identificar cuáles de éstas tienen una correlación con conceptualizaciones como la de desarrollo territorial. De acuerdo con lo que plantea Madoery (2008: s/p), las connotaciones de territorio implican:

Una superficie simbolizada, dotada de significado a partir de los procesos sociales diversos que en éste se expresan. Un entorno en el cual se fraguan las relaciones sociales y económicas, terreno de interacciones múltiples y constitución de actores (...) Un espacio de intervención, de ordenamiento, de vertebración; y fundamentalmente, un territorio proyectado, un espacio de construcción, el lugar del "proyecto político de desarrollo" y un factor estratégico de oportunidades de desarrollo.

Las ideas relacionadas con el desarrollo, entonces, ocupan un lugar preponderante en la orientación de las políticas públicas; al haberse convertido en una "idea-fuerza" se dejaron de lado las discusiones sobre lo que conlleva el ideal del desarrollo y de cómo fue la génesis de este concepto y cuáles son sus implicancias. De todas maneras, y a efectos prácticos de este trabajo, no se hará un recorrido exhaustivo de los debates en torno a la cuestión del desarrollo; por el contrario, el análisis se enfocará en identificar cómo fue que las preocupaciones por el territorio cobraron relevancia en el INTA como un eje vertebrador de sus intervenciones en el espacio. Analizar la incorporación de un concepto como el de territorio permite indagar cuál es la relación que el mismo tiene con preocupaciones institucionales muy arraigadas en el INTA, como los procesos de desarrollo regional. En términos de Albadalejo: 
Hoy en día se habla del territorio de los actores, entonces el trabajo del desarrollo territorial tiene que ver con delimitar, para cada acción, el territorio de intervención desde lo cotidiano (...) El INTA nace con una idea de transferencia de tecnología. En INTA la agricultura era un proyecto territorial también. Antes de que exista la palabra, en otros periodos ya se la usa mucho (Albadalejo, entrevista, 20 de febrero de 2017).

Esto que menciona Albadalejo (2017) acerca de la delimitación conceptual de territorio se puede pensar a la luz de la renovación conceptual que se revela en los estudios geográficos (en particular de la geografía rural) a partir de los 80 y 90, cuando surge una necesidad de incorporar nuevas perspectivas teóricas, especialmente de la economía política. En este marco, uno de los ejes a abordar en esta investigación es el análisis sobre el rol que desempeña la administración pública en la configuración del espacio rural (García-Ramón, 1995). Esto se vincula con el gran despliegue que ha cobrado en los últimos años el concepto de Desarrollo Territorial Rural (en adelante DTR), que se popularizó en América Latina a partir del reconocimiento de que las estrategias de desarrollo rural tradicionales no habían podido reducir la pobreza rural (Manzanal et al., 2009). Particularmente el DTR implica "que los territorios rurales logran 'desarrollarse' cuando consiguen posicionarse en mercados dinámicos, cohesionan a sus propios actores y se articulan con otros actores y territorios rurales $\mathrm{y} / \mathrm{o}$ urbanos" (Manzanal et al., 2009: 133).

Tampoco se puede soslayar la influencia que ejercen en los espacios rurales actores que se encuentran por fuera del ámbito local: desde organismos supranacionales hasta empresas transnacionales que llevan adelante estrategias globales, pero localizadas territorialmente. La relación que tiene el concepto de territorio con estas dinámicas espaciales y temporales fue analizada por Santos (1993) con sus nociones de "horizontalidades y verticalidades". Esto nos permite pensar en dinámicas de transformación territorial que se ven atravesadas por la influencia de factores exógenos, así como también por dinámicas que están fuertemente enraizadas o regional o localmente.

El INTA aparece, en este sentido, como un actor de gran centralidad cuando se trata de trazar, influir y generar políticas e instrumentos programáticos de gestión que puedan lidiar con enorme heterogeneidad con actores, intereses y disputas de poder involucradas. Sobre todo al considerar que el territorio, a nivel conceptual, deviene en un campo fuertemente político porque se vislumbra como un escenario de intervención para el desarrollo (Schejtman y Berdegué, 2004). 


\subsection{La circulación de los conceptos de territorio en Argentina}

Las distintas conceptualizaciones referidas al territorio circularon en Argentina y sirvieron como marco conceptual para la génesis de políticas públicas enfocadas en fomentar procesos de desarrollo social y económico en instituciones como el INTA.

Siguiendo a Cicalese (2014) es posible reconstruir una genealogía sobre la influencia de la cultura francesa en el sistema político y cultural argentino, la cual comienza a tener un impacto muy grande ya desde principios del siglo XIX. Esta proyección cultural e ideológica fue decisiva en distintos ámbitos del país (desde el derecho, la literatura, hasta las ciencias sociales) y, particularmente, en todas las cuestiones relacionadas con el abordaje de las problemáticas espaciales. La geografía francesa, en sus distintas variantes, tuvo mucho peso en la conformación del campo disciplinar de la geografía argentina y, a su vez, en las teorías, métodos y selección de problemas a ser abordados por los gestores de las políticas públicas a distintos niveles. (Cicalese, 2014)

La circulación franco-argentina de conceptos y metodologías de acción espacial ejerció una influencia muy significativa en distintas instancias estatales, particularmente, en instituciones que tenían un mandato de acción territorial específico. A partir de convenios de intercambio y articulación académicos entre la Universidad de Burdeos y la Universidad Nacional de Cuyo comenzaron a llegar a Argentina académicos y estudiantes franceses que realizaron trabajos de investigación aplicada en el país. En ese contexto y aunado a una acción diplomática francesa muy activa por promover modelos de desarrollo en los países subdesarrollados, fue como se realizaron estudios de geografía aplicada en Argentina. Esta política de difusión cultural francesa se desarrolló con intensidad en el ámbito universitario y promovió la formación no sólo de estudiantes o académicos, sino también de dirigentes y mandos medios en instituciones como el INTI, el CONICET y el INTA.

Es importante destacar, en el marco de esta circulación de conceptos y metodologías de abordaje de las problemáticas espaciales, el rol que desempeñaron ciertos actores en esos procesos de difusión. Una figura de gran centralidad a partir de la década de 1960 es la del geógrafo francés Romain Gaignard. Como reseña Cicalese (2017), Gaignard pasó casi toda la década de 1960 en Argentina, donde escribió su tesis doctoral denominada "Estructuras agrarias pampeanas" de la cual se desprendería su afamada publicación La pampa argentina: ocupación-poblamiento-explotación de la conquista a la crisis mundial 1550-1930. Este y otros trabajos que publicó en la época cimentaron un gran prestigio académico y lo 
llevaron a convertirse en uno de los referentes de la conformación del campo disciplinar de la geografía en la Universidad Nacional de Cuyo. De acuerdo con Cicalese (2017: 13):

Los geógrafos franceses, como hombres de la $5^{\circ}$ República, manejaban una ciencia carrefour muy conveniente en el plano de las relaciones políticas internacionales en momentos en que la Argentina planteaba un modelo de desarrollo nacional donde los agentes externos provenientes del campo económico en asociación con los estados eran pensados como socios clave para lograr el despegue.

El creciente prestigio académico de Gaignard lo llevó a consolidar el establecimiento de redes académicas entre Francia y Argentina, y a tener una cierta gravitación política a partir de estas vinculaciones. En Argentina, la figura de Romain Gaignard como geógrafo diplomático fue muy importante en esa construcción de redes transoceánicas, vinculándose con colegas de distintas escuelas geográficas e históricas (Cicalese, 2017). Uno de sus interlocutores habituales de la época fue Horacio Giberti, quien fue nombrado como Secretario de Agricultura y Ganadería durante el tercer gobierno de Perón y había sido uno de los fundadores y segundo presidente del INTA (entre 1958 y 1961). Albadalejo (2017) al respecto de esto señala:

Lo de Gaignard pasó en la época de Frondizi, una época desarrollista [...] se quedó 9 años en Argentina y tuvo mucho vínculo con el INTA. Esto quedó frustrado por la cuestión política, con el golpe militar. La idea desde el INTA era introducir la idea de desarrollo rural y la idea de que el desarrollo de la actividad agropecuaria es indisociable del desarrollo del territorio (Albadalejo: entrevista, 20 de febrero de 2017).

La participación de geógrafos franceses fue central en este primer periodo de profesionalización de la geografía argentina, que se orientaría así a convertirse en una "geografía aplicada" con fuertes vinculaciones con distintas instituciones estatales que tenían mandatos de acción espacial como el INTA. Estos procesos de difusión cultural llevados adelante por instituciones francesas lograron crear en el ámbito de la geografía argentina una línea epistemológica con una fuerte raigambre regional que se terminaría consolidando. De la mano de la fortaleza disciplinar de la geografía francesa se desarrollaría entonces una escuela geográfica en Argentina con una visión regional y con un compromiso de apoyar el desarrollo del país a través del estudio y las políticas de intervención territorial.

Si bien los procesos de colaboración académica que había impulsado Gaignard fueron interrumpidos a finales de la década del 60 y durante los 70, por cuestiones políticas a partir de los distintos golpes militares, a su vuelta a Francia este investigador tomó cargos institucionales en la 
Universidad de Toulouse y ayudó a la emergencia de un grupo muy fuerte en geografía rural (formados por un geógrafo social llamado Bernard Kayser), quien tendría una influencia perdurable en el campo disciplinar de la geografía rural argentina (Sol, 2015).

Algunos investigadores franceses, luego del retorno de la democracia, retomaron su colaboración con Argentina, y en particular con el INTA. Uno de estos investigadores que se constituyó en un actor central de esta dinámica de intercambio académica y conceptual es Christophe Albadalejo, que llegó a Argentina en 1982 para realizar una tesis de doctorado en geografía para la Universidad de Grenoble y comenzó a trabajar en cuestiones de desarrollo territorial y agricultura familiar en la provincia de Misiones. Su cooperación con el INTA comenzó en 1984 y desde ese lugar propuso que se retomara el trabajo con el Institut National de la Recherche Agronomique (INRA), el cual se había desarticulado durante la última dictadura cívico-militar (Albadalejo, entrevista, 20 de febrero de 2017).

Estas dinámicas de cooperación franco-argentinas continúan hasta la actualidad: Albadalejo es el director francés del instituto de cooperación denominado Laboratorio Agriterris. ${ }^{2}$

\section{Implementación del enfoque territorial: las políticas públicas y la creación de los PRET}

No se puede soslayar que la formulación de toda política pública va de la mano de una definición explícita o implícita de una serie de elementos normativos que se encuentran alineados a un proyecto político o a una visión de un futuro deseable (Mosca, 2015). Es en este sentido que se tomarán los aportes desarrollados por Muller (2006: 95):

\footnotetext{
Las políticas públicas son el lugar donde la sociedad construye su relación con el mundo [...] entonces las políticas deben ser analizadas como los procesos a través de los cuales son elaboradas las representaciones que tiene una sociedad para comprender y actuar sobre los problemas de la realidad que percibe como tales.
}

Los distintos actores crean su percepción sobre el problema de acuerdo con esa imagen cognitiva, y a los términos en los cuales dicho problema fue planteado. Cuando se habla de la puesta en función de una política pública

${ }^{2}$ A partir del 2007 esta institución congregó a cuatro instituciones francesas (INRA, Universidad de Toulouse Le Mirail, CIRAD y Montpellier SupAgro), a cuatro instituciones argentinas (Universidades Nacionales de La Plata, Mar del Plata y del Sur y el INTA) y a una brasileña: la Universidad Federal de Pará (UFPA). Albadalejo, a su vez, fue partícipe de la formación de muchos investigadores argentinos cuyos aportes científicos han sido fundamentales para el INTA. 
específica, la misma se operacionaliza a través de instrumentos. Se parte de la base de considerar que los instrumentos de política se conciben como un tipo ideal que viene a agrupar distintas iniciativas disímiles, que son utilizadas de manera combinada por los responsables de gestionar las políticas públicas.

Como señala Velasco (2007: 1): "cada instrumento responde a una naturaleza, objetivos y carácter propio, y es su uso alternativo o simultáneo el que permitiría conocer estrategias concretas de las políticas". En el análisis de políticas públicas se estudian los instrumentos, con el fin de comprender cuál es el vínculo que hay entre la formulación y la operacionalización de una política pública determinada. En este sentido, la génesis de la política institucional que creó los PRET se puede rastrear a partir de la recuperación de la democracia ( $\mathrm{y}$ fundamentalmente a mediados de los años 80), cuando se comenzaron a gestar diversas modificaciones burocráticas y de políticas internas en el INTA que tuvieron grandes repercusiones en la gestión institucional del organismo. Según la página oficial del INTA:

La descentralización se concretó principalmente en la constitución de los 15 Consejos de Centros Regionales y 3 Consejos de Centros de Investigación, a los cuales se les confirió la responsabilidad de determinar prioridades y distribuir los fondos en cada una de sus jurisdicciones. Estos Consejos se conformaron con representantes de distintas asociaciones de productores, de los gobiernos provinciales, de la comunidad científica y de las universidades (INTA, 2019).

A su vez se produjo el establecimiento de un instrumento de gestión denominado Proyectos Regionales (en adelante PR). Estos últimos fueron el antecedente más cercano a los PRET, y constituían un instrumento de gestión que tenía por objetivo transferir el conocimiento generado por la institución en los centros regionales mencionados en el apartado anterior. Ya desde este momento se observa cómo la cuestión territorial comenzó a ser de mayor trascendencia en la gobernanza del INTA.

A partir de estas modificaciones, surgidas originalmente a mediados de los años 80, la acción espacial del INTA se ejecutaba mediante dos modalidades: los Programas Nacionales (en adelante PN), orientados por cadenas productivas, y los $\mathrm{PR}$, que estaban más relacionados con la extensión y la transferencia. Esta "doble vía" por la cual se canalizaban los recursos y los esfuerzos institucionales implicó una multiplicidad de iniciativas existentes para intervenir territorialmente que llevó a una dispersión importante de los recursos invertidos para dar respuesta a problemáticas regionales y locales.

A la par que se iban desenvolviendo los mencionados Proyectos Regionales, y ya instalados muy fuertemente los programas de intervención 
(ProFeDer, ProHuerta, Cambio Rural ${ }^{3}$ ), en el Plan Estratégico Institucional (PEI) 2005-2015, lanzado en 2004, se institucionalizó el enfoque territorial. La adopción de este enfoque implicó reconocer la importancia de una mirada más sistémica a la hora de atender los problemas de desarrollo que se encontraban presentes en cada espacio geográfico; en este sentido, las experiencias generadas en el devenir de los distintos programas de intervención fueron importantes para el aprendizaje institucional que llevaría a ver la necesidad de repensar la manera en la cual el INTA se vinculaba con los territorios.

Nuevamente en este caso se puede vislumbrar la creciente importancia que, en términos de Muller (2006: 95) tuvo el concepto de territorio como un eje vertebrador de las respuestas institucionales a "los problemas de la realidad que percibe como tales". Sobre todo teniendo en cuenta que esto se materializó en un contexto político nacional en el cual se lanzaron una cantidad importante de programas e instrumentos públicos para atender una demanda creciente del poder político (y en algún sentido de la sociedad en su conjunto), por promover la vinculación de la ciencia y la tecnología con la sociedad; específicamente, se buscó generar instancias de vinculación que permitieran acercar a los productores de conocimiento con sus potenciales usuarios, en un marco de un régimen de ciencia estratégica (Rip, 2002), en el cual el desarrollo social y la inclusión, particularmente a partir de la gestión gubernamental nacional que llegó al poder en el 2003 (y continuaría hasta 2015) pasaron a ser principios rectores de las políticas de ciencia y tecnología.

\subsection{La operacionalización del enfoque territorial: puesta en función de los PRET}

La aplicación de este enfoque territorial que surgió del PEI 2005-2015 fue muy dispar en los distintos Centros Regionales (en adelante CR) del país; sin embargo en el CR Tucumán-Santiago del Estero se comenzó a trabajar fuertemente en este sentido, y se promovió orientar los esfuerzos institucionales hacia acciones que tuvieran impacto territorial y que, por consiguiente, todas las actividades de investigación y extensión se diagramaran en función de esa dimensión. Fue así que surgió una primera experiencia en la cartera de proyectos 2005-2009, en la cual este CR reorientó sus Proyectos Regionales para que se constituyeran en el principal instrumento

\footnotetext{
${ }^{3}$ Algunos de los principales programas de intervención territorial son la Unidad de Planes y Proyectos de Investigación y Extensión para Pequeños Productores, conocida posteriormente como Unidad de Minifundio; el Programa Federal de Reconversión Productiva para las Pequeńas y Medianas Empresas Agropecuarias (Cambio Rural); el Programa Pro-Huerta; el Programa para Productores Familiares (Profam); y el Programa Federal de Apoyo al Desarrollo Rural Sustentable (Profeder).
} 
de aplicación del enfoque territorial. En el CR Tucumán-Santiago esta política impulsada por su director implicó dejar de concebir a los viejos PR en su orientación por cadena productiva, y pasar a orientarlos en una nueva visión más alineada con las problemáticas locales que excedían a lo netamente productivo. Cómo señala una investigadora:

En el 2004 se hace la elaboración del PEI y ahí se definen los lineamientos para el 2015. Uno de los lineamientos era implementar el enfoque territorial, pero algunos centros regionales no lo tomaron en consideración. Los de Tucumán y Santiago del Estero insistían que el foco tenía que estar en los territorios [...]. Ellos forzaron a la institución cumplir el PEI, dijeron: tiene que ir por acá. La mayoría de los otros centros siguieron con la modalidad anterior, pero ellos cambiaron. El Director de Tucumán y Santiago del Estero fue luego Director Nacional del INTA, Eliseo Monti. Cuando pasa a ser Director Nacional lo pide para todos los Centros regionales. Yo pienso que lo impulsó porque el viene de la extensión, conoce el territorio, lo que pasa allí y conoce lo que pasa en la I+D. Siempre se criticó el divorcio entre I+D y transferencia. Esto era la oportunidad de juntarlos (Anónimo 8, entrevista, 19 de mayo de 2017.)

En este sentido, es importante destacar la formación profesional y la trayectoria del impulsor de estas iniciativas: el director de este CR, Eliseo Monti tenía una experiencia amplia en gestión, y se había formado en una Maestría en Desarrollo Económico de América Latina en España, en la cual había trabajado temas relacionados con desarrollo local y territorial, aplicando marcos teóricos provenientes de las teorías de sistemas complejos. En sus palabras:

Yo vengo de la extensión, laburé toda la vida en contacto con la gente (...) Nosotros nos formamos en España: ¿por qué? Porque ahí estaban aquellos que más circulaban en términos de desarrollo local. Hicimos una maestría allá, le pusimos marco teórico a la realidad. Ahí se cruzan las cosas, el hecho de estar en la gestión, estuve casi 6 ańos en Cambio Rural, anduvimos en todo el país, haciendo monitoreos. (Monti, videoconferencia, 6 de noviembre de 2017).

Monti concursó y ganó el cargo de director nacional del INTA en 2011. Una vez en ese cargó decidió avanzar en la implementación, a nivel nacional, de la experiencia que había desarrollado en su anterior cargo de director de CR. Los PRET, entonces, pasaron a constituirse en un instrumento de política pública orientado a abordar la complejidad territorial, fomentar la innovación y atender las demandas sociales en todo el INTA a nivel nacional.

De acuerdo con lo que se plantea en distintos documentos institucionales del INTA (2004; 2016), el principal objetivo de los PRET fue promover procesos de innovación en el territorio para contribuir al desarrollo de los 
actores y sistemas productivos presentes en la región. De esta manera los PRET se constituyeron en una estrategia de gestión de las intervenciones territoriales del INTA, que buscó abordar las problemáticas a través de la acción de los cinco componentes estratégicos del INTA (Investigación y Desarrollo Tecnológico; Transferencia y Extensión; Relaciones Institucionales; Comunicación y Vinculación) en conjunto con los actores presentes en el espacio geográfico delimitado por cada proyecto. Estos cinco componentes estratégicos se dividen en dos componentes identitarios y tres articuladores:

Componentes identitarios: Investigación y Desarrollo, para generar conocimientos en los principales ejes de innovación del SAAA anticipando y abordando sus demandas, necesidades y oportunidades, y de acuerdo con las exigencias que emanan de un contexto global en permanente cambio y redefinición. Extensión y Transferencia, para contribuir a la dinamización de espacios para el intercambio de información, conocimientos y tecnologías que impulsen procesos de innovación, respondiendo a las necesidades de un territorio como construcción social, identificando las oportunidades de transformación e incorporando los saberes e intereses locales para el desarrollo sostenible (INTA, 2016: 37).

Componentes articuladores: Vinculación Tecnológica, que concreta la asociación con los sectores público y privado y expanda las oportunidades de desarrollo productivo y social regional a través de diferentes modalidades de alianzas. Relaciones Institucionales, para fortalecer los nexos de cooperación entre los actores públicos y privados a los fines de ampliar las oportunidades de generación de conocimiento e innovación en los niveles nacional, regional, local e internacional. Información y Comunicación, para diseñar la arquitectura y gestionar de manera inteligente la información que impulse el conocimiento y la innovación, fortaleciendo el flujo de la comunicación en la organización, sus entornos y en los territorios (INTA, 2016: 38).

La aplicación de los PRET se generalizó en la institución durante el 2012, y en la cartera de proyectos aprobada en 2014 se lanzaron formalmente 122 PRET (figura 1) (INTA, 2016). A partir de esta cartera de proyectos, los PRET se convirtieron oficialmente en un eje transversal a través del cual se identificaron los problemas, se articularon las respuestas institucionales, se asignaron los recursos y se difundieron los resultados. Los PRET se concibieron como plataformas articuladoras y canalizadoras de recursos, conocimientos y oportunidades, orientadas a contribuir a través de innovaciones tecnológicas y organizacionales al aprovechamiento de las oportunidades y a la resolución de los problemas en cada área geográfica (INTA, 2016).

Los PRET se delimitaron espacialmente de acuerdo con un criterio de áreas geográficas homogéneas; sin embargo, y a efectos de simplificar la gestión a nivel administrativo y burocrático, algunos PRET no sólo se 


\section{Figura 1}

\section{Territorio argentino dividido en 122 PRET}

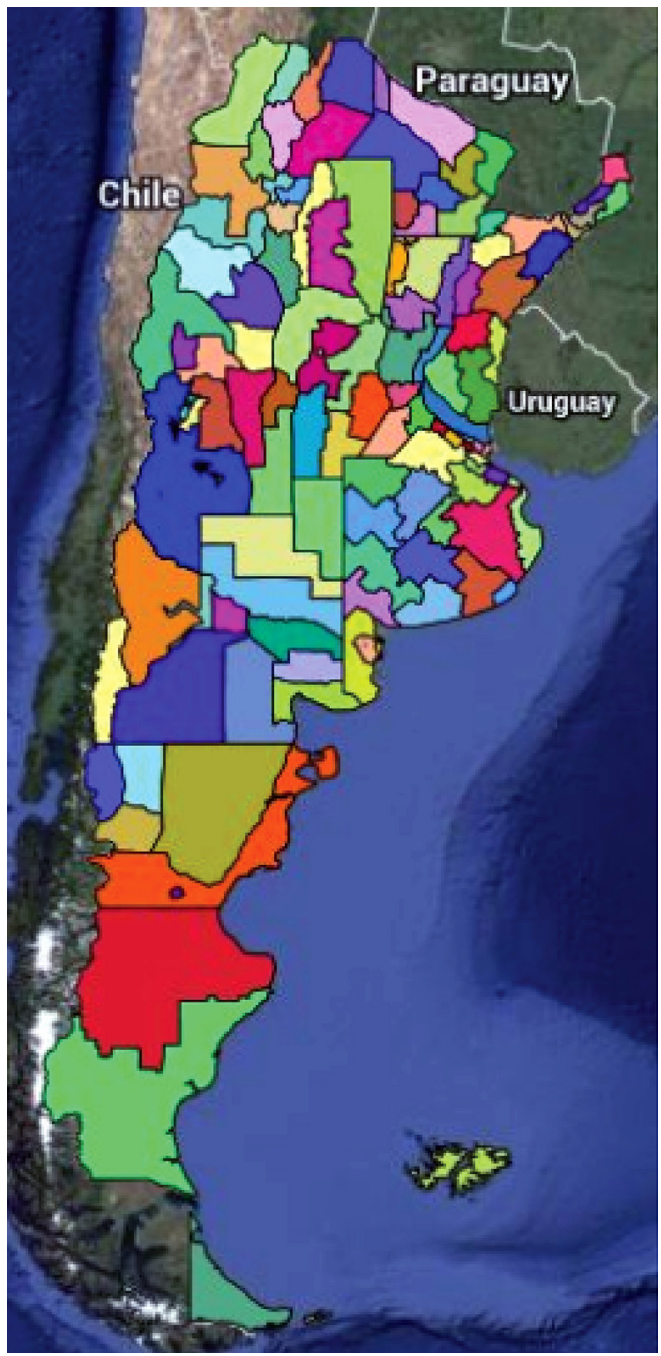

Fuente: Instituto Nacional de Tecnología Agropecuaria (INTA, 2014).

delimitan por homogeneidad geográfica, sino también por criterios de demarcación más políticos (Anónimo 2, entrevista, 20 de febrero de 2017). A nivel operativo fueron pensados para ser instrumentados mediante la conformación de un Equipo de Gestión (en adelante EG), que es el encargado de llevar adelante todas las actividades propuestas. Este EG incluye la figura de un coordinador, los jefes de las Agencias de Extensión Rural (AER) del área y los jefes (o bien, los referentes temáticos) de los distintos 
grupos de investigación que están trabajando temáticas relacionadas con las problemáticas que aborda el PRET. También participan en el EG representantes del Programa Federal de Apoyo al Desarrollo Rural Sustentable (ProFeDer) y agentes de Cambio Rural, así también como referentes del ProHuerta (INTA, 2016).

La modalidad de gestión implica la realización de reuniones con cierta periodicidad en las cuales se discuten las líneas de acción, las estrategias para llevar adelante las actividades, la priorización en la utilización de los recursos financieros y el reparto y ejecución del presupuesto. De acuerdo con Monti: "Esto no es un proyecto de investigación es un proyecto de gestión. Es el único lugar donde se junta todo, comunicación, extensión, investigación" (entrevista, 6 de noviembre de 2017). Las acciones ejecutadas por cada PRET surgieron de procesos de diagnóstico participativo, en los cuales se buscó garantizar el acceso de los actores presentes en el territorio que cuenten con una problemática a ser resuelta en el marco del proyecto.

\title{
2.2. Los PRET: controversias sobre su implementación
}

Uno de los principales objetivos de los PRET fue avanzar en la articulación de la investigación con las demandas reales de los territorios, es decir, a nivel institucional se buscó que la definición de los temas a investigar tuviera una correlación más intensa con las problemáticas reveladas en los territorios a través de la participación de los distintos actores en la plataforma de los PRET. Esto generó controversias entre los investigadores que, en su mayoría, se manifestaron en contra de modificar sus líneas de trabajo y abandonar sus áreas de expertise para trabajar a partir de problemáticas y demandas que surgían de los territorios. En palabras de un investigador, cuyo trabajo está más orientado a la ciencia básica:

\begin{abstract}
A nivel jerárquico tuvieron que tomarlo porque fue una bajada de línea, pero fue controversial en todo momento. Lo que hicieron fue adaptarse: lo aceptaron pero no cambiaron sustancialmente la manera de trabajar. En (la sede de) INTA Castelar te dicen: yo no tengo nada que ver con los PRET: "lo que hago yo no es de interés para ningún problema regional” (Anónimo 3, entrevista, 22 de febrero de 2017).
\end{abstract}

Similarmente otra investigadora participante de un Programa Nacional comentó:

En realidad es más habitual de lo que uno piensa. Continuar con la línea que se trabaja eternamente. Es una inercia muy difícil de romper. tb son lugares de poder. Soy un referente de este tema y de repente tengo que cambiar (Anónimo 4, entrevista, 25 de mayo de 2017). 
Por otro lado, a partir de las entrevistas, se identificó un sector de investigadores para los cuales la implementación de los PRET les fue de utilidad para incorporar una perspectiva territorial en sus quehaceres laborales de investigación. De acuerdo con una investigadora:

Con los que conversé me dijeron que les ayudó mucho en cuanto a la mirada. Por más que estés en tu laboratorio haciendo tal cosa, tenés presente lo que estuviste hablando en una reunión en el territorio. En el día a día no les cambia a los investigadores sus objetivos de investigación... Lo que dicen es eso, como que cambia la visión de para qué están trabajando... y que es positivo. (Anónimo 8, entrevista, 19 de mayo de 2017).

Es importante agregar que la gran mayoría de los proyectos de investigación previos a la llegada de los PRET eran (en su gran mayoría) gestionados y coordinados por investigadores; una vez lanzados los PRET, pasaron a ser coordinados por extensionistas. De acuerdo con el coordinador Nacional de Transferencia y Extensión, Diego Ramilo (entrevista, 20 de octubre de 2017):

La mayoría de los PRET tienen un liderazgo de los extensionistas. Los PRET dieron jerarquía y rango a ese rol de articulador. Muchos de esos puestos fueron ocupados por extensionistas (...) Permitieron generar los mecanismos para discutir el hecho de que hacemos investigación y extensión en el marco de la innovación en los territorios.

Según una participante del Programa Nacional para el Desarrollo y la Sustentabilidad de los Territorios: "la mirada más crítica de la gestión de los PRET fue de parte de los investigadores, surgen distintas cosas. Está bueno analizar la dimensión del poder. Hace ruido porque el presupuesto pasa a ser manejado por los PRET" (Anónimo 9, entrevista, 7 de diciembre de 2016).

Siguiendo lo planteado por Elzinga y Jamison (1996) se observa claramente dentro de INTA que la tensión entre investigación y extensión se asimila a una competencia que surge de la interacción dinámica que se produce entre actores que terminan conformando distintas culturas políticas: el sector de los investigadores, con una cultura académica orientada a preservar la autonomía, la integridad, la objetividad, y el sector de los extensionistas, con una cultura económica vinculada al sector de la gestión, que pone el foco en el uso y la aplicación de la tecnología.

Es necesario aclarar que estas culturas políticas constituyen un tipo ideal que, como tal, no se presenta necesariamente de forma pura: dentro del grupo de los investigadores se encuentran actores que van a promover una interacción mayor con los actores económicos o sociales; mientras que en el grupo de los extensionistas se pueden observar actores que tienen una 
cultura cívica o burocrática en términos de lo planteado por Elzinga y Jamison (1996).

El sector de extensión rápidamente tomó la iniciativa de los PRET como una ventana de oportunidad para conseguir un mayor capital simbólico, a través de la posibilidad de acceder a mejores cargos y de tener una mayor incidencia en las decisiones de construcción de agendas de investigación y extensión territoriales. En contraposición con esto, la mayoría de las oposiciones a los PRET provino de los directores de centros de investigación e institutos, así como de investigadores que tenían líneas de trabajo muy consolidadas, los cuales vieron amenazada su posición, y las posibilidades de contar con financiamiento, a partir del cambio en la determinación de las agendas de investigación y en el manejo de los recursos de financiamiento. La determinación de las disputas de poder en relación con quien define las agendas de investigación deviene un asunto central para el análisis de la implementación de los PRET. En relación con esto, una investigadora de la Estación Experimental Agropecuaria Área Metropolitana de Buenos Aires afirmó (2017):

En INTA el peso de sostener lo que existe se come todo el presupuesto, entonces no hay presupuesto para repensar temas críticos y trabajar en algo más focalizado y no tan abierto. El desafío es cómo definir eso... esa disputa es crítica. Es tan crítica que ya no sé si la pregunta es esa... ¡̇es posible definir una agenda? Cómo hacés sin que eso suene a imposición, que es lo que pasó con esto de los PRET. Muchos investigadores lo sintieron como una imposición. Pero por buena voluntad tampoco va a funcionar (Anónimo 1, entrevista personal, 24 de mayo de 2017).

Consultado por su evaluación acerca de la incidencia que tienen los PRET para definir las agendas de investigación, Eliseo Monti (2017) comentó:

El impacto en las agendas de investigación es bastante heterogéneo. Depende con quién hables, hay gente que no le interesa que sea así. Hay investigadores que nunca en la vida salen de las estaciones experimentales. Es un cambio de estrategia, de visión. Hay gente que piensa que el INTA sólo tiene que hacer investigación. El desarrollo lo tiene que hacer desarrollo social... pero son visiones. (Eliseo Monti, videoconferencia: 6 de noviembre de 2017).

Esta heterogeneidad que menciona Monti remite a las dificultades que implica traducir en una nueva agenda de investigación la voluntad de los distintos grupos por aumentar la utilidad social de sus investigaciones. Fue así que la instrumentación de los PRET terminó siendo bien recibida por sectores del INTA que tenían algo más desarrolladas estrategias de generación de conocimiento en conjunto con otros actores sociales. De 
acuerdo con el director del Centro de Investigación de Agroindustria del INTA (2017):

\begin{abstract}
Para mí lo de los PRET está muy bien, porque hay que mirar esa demanda local, que están armando proyectos de acuerdo a la demanda local de la región, y después lo suban a los investigadores y la lleven a los centros con capacidades más sofisticadas. Eso tiene que ser así, es muy bueno. Si estamos bien articulados, yo creo que está muy bueno. (Anónimo 5, entrevista, 23 de febrero de 2017)
\end{abstract}

Sin embargo, en centros de investigación y grupos que no tenían tradición por articularse con esos actores, la llegada de los PRET los llevó a cuestionarse el grado de relevancia y pertinencia de su trabajo, así como vislumbrar una necesidad creciente de generar capacidades que les permitieran establecer redes de relaciones con agentes externos y posibles demandantes de su producción científico-tecnológica.

\title{
Conclusiones
}

En este trabajo se puede observar un proceso muy dinámico de circulación de ideas, conceptos y maneras de gestionar el espacio que surgieron a partir de las distintas redes de cooperación académicas entre Francia y Argentina. Esas redes se consolidaron a lo largo del tiempo, institucionalizándose de manera creciente en Argentina y permitiendo que ciertos conceptos como el de territorio o el de enfoque territorial fueran tomados como ejes vertebradores de las políticas institucionales en relación con la gestión de la investigación y la extensión dentro de instituciones como el INTA.

Este enfoque territorial, una vez adoptado como una política institucional de gran centralidad, fue operacionalizado en el INTA a través de una serie de instrumentos de gestión, siendo una de sus últimas formas los PRET. A partir del 2014 (y hasta el 2018, cuando finalizaron) los PRET se convirtieron en la principal política de la institución para identificar las demandas, gestionar los recursos y generar las respuestas a las problemáticas detectadas en los territorios.

El lanzamiento de los PRET fue eje de controversias bastante extendidas dentro de la institución. Los investigadores más orientados a hacer ciencia básica estuvieron mayormente en contra, mientras que los investigadores que estaban más orientados a hacer ciencia aplicada tuvieron una visión en general más positiva de la institucionalización de los PRET.

El surgimiento de los PRET se puede pensar, en este contexto, como el emergente de un nuevo balance de poder interno del INTA en el cual la función de extensión comenzó a recuperar centralidad en la operacionalización de las políticas institucionales, utilizando el concepto de terri- 
torio como un paraguas conceptual que brindó legitimidad a la manera en la cual se conformaban las agendas de investigación y extensión dentro de INTA y se asignaban los recursos financieros que se invertían en los territorios. A su vez, los PRET permitieron articular una serie de políticas e instrumentos del INTA que se encontraban dispersos bajo una misma herramienta programática, la cual contribuyó a alinear a la institución a un contexto político y social del país que demandaba un rol más activo de las instituciones de ciencia y tecnología en la resolución de las problemáticas socioeconómicas y espaciales.

\section{Fuentes consultadas}

Albornoz, Mario (2015), "Cambio tecnológico y cultura institucional: el caso del INTA", Revista Iberoamericana de Ciencia, Tecnología y Sociedad, 10 (29), Buenos Aires, REDES/Centro de Estudios sobre Ciencia, Desarrollo y Educación Superior, pp. 41-64.

Alemany, Carlos (2003), “Apuntes para la construcción de los periodos históricos de la extensión rural del INTA", en Ricardo Thorntorn y Gustavo Cimadevilla (comps.), La extensión rural en debate, Buenos Aires, Ediciones INTA, pp. 137-172.

Argentina.gob.ar (2020) “Decreto/Ley 21680/1956”, 4 de diciembre de 1956, Buenos Aires, Poder Ejecutivo Nacional, <https:/www. argentina.gob.ar/normativa/nacional/decreto_ley-21680-1956$77763 /$ texto $>20$ de mayo de 2020.

Cicalese, Guillermo (2017), "La invención de un programa y una comunidad disciplinaria en la Universidad Nacional de Cuyo. Geógrafos visitantes y textos franceses para una ciencia carrefour en la segunda mitad del siglo XX", ponencia presentada en las XII Jornadas Cuyanas de Geografía, 20 al 22 de septiembre, Ciudad de Mendoza, Universidad Nacional de Cuyo, <http://nulan.mdp. edu.ar/2754/1/cicalese-2017.pdf>, 3 de diciembre de 2019.

Cicalese, Guillermo (2014), "Diplomacia de ideas, política académica regional y Geografía. Una ciencia francesa para narrar e intervenir el territorio argentino de la Región Cuyana a la Pampa Gringa, 1947-1973", Scripta Nova. Revista Electrónica de Geografía y Ciencias Sociales, 18 (465), Barcelona, Universidad de Barcelona. 
Correia de Andrade, Manuel (2002), "Territorialidades, desterritorialidades, novas territorialidades: os limites do poder nacional, e do poder local”, en Milton Santos, María Adélia Souza, Maria Laura Silveira (org.), Território: globalização e fragmentação, São Pablo, Hucitec, pp. 213-220.

Elzinga, Aant; Jamison, Andrew (1996), "El cambio de las agendas políticas en ciencia y tecnología”, Revista Zona Abierta, núms. 75-76, Madrid, Fundación Pablo Iglesias, pp. 91-132.

García-Ramón, María Dolors (1995), Geografía Rural, Madrid, Síntesis.

Gárgano, Cecilia (2013), "Ciencia y Dictadura: producción pública y apropiación privada de conocimiento científico-tecnológico. Dinámicas de cooptación y transferencia en el ámbito del Instituto Nacional de Tecnología Agropecuaria (INTA) durante la última dictadura cívico-militar argentina (1976-1983)", Crítica y Emancipación, núm. 10, Buenos Aires, CLACSO, pp. 135-175.

Harvey, David (1990), La condición de la posmodernidad. Investigación sobre los orígenes del cambio cultural, Buenos Aires, Amorrortu Editores.

InfoLEG (Información Legislativa) (1956), “Decreto/Ley 21680/1956”, 4 de diciembre de 1956, Buenos Aires, Poder Ejecutivo Nacional, $<$ http://servicios.infoleg.gob.ar/infolegInternet/verNorma. do?id=77763>, 5 de diciembre de 2019.

INTA (Instituto Nacional de Tecnología Agropecuaria) (2019), "Historia del INTA", Buenos Aires, Instituto Nacional de Tecnología Agropecuaria, <https://inta.gob.ar/paginas/historia-del-inta>, 5 de febrero de 2020.

INTA (Instituto Nacional de Tecnología Agropecuaria) (2017), “AGRITERRIS: Un dispositivo internacional de investigación-formación sobre el desarrollo territorial en áreas rurales", Buenos Aires, Instituto Nacional de Tecnología Agropecuaria, <https://inta.gob. ar/documentos/agriterris-un-dispositivo-internacional-de-investigacion-formacion-sobre-el-desarrollo-territorial-en-areas-rurales>, 3 de diciembre de 2019.

INTA (Instituto Nacional de Tecnología Agropecuaria) (2016), "Marco conceptual para la implementación del enfoque territorial”, Buenos Aires, Instituto Nacional de Tecnología Agropecuaria, <http:// 
inta.gob.ar/sites/default/files/marco_conceptual_para_la_implementacion_del_enfoque_territorial.pdfs, 3 de diciembre de 2019.

INTA (Instituto Nacional de Tecnología Agropecuaria) (2014), "Los mapas de los PRET's en un solo click", INTA, 17 de marzo de 2014, <https://inta.gob.ar/noticias/los-mapas-de-lospret\%C2\%B4s-en-un-solo-click>, 3 de diciembre de 2019.

INTA (Instituto Nacional de Tecnología Agropecuaria) (2004), "El INTA que queremos. Plan Estratégico Institucional 2005-2015”, Buenos Aires, Instituto Nacional de Tecnología Agropecuaria, <http:// inta.gob.ar/sites/default/files/script-tmp-plan_estrategico_ inta_2005-2015_.pdf>, 3 de diciembre de 2019.

Lopes de Souza, Marcelo José (1995), "O territorio: sobre espaço e poder, autonomia e desenvolvimento", en Iná Elias de Castro, Paulo Cesar da Costa Gómez y Roberto Lobato Correa, Geografia: conceitos e temas, Río de Janeiro, Ed. Bertrand, pp. 77-116.

Madoery, Oscar (2008), “Conceptos básicos del desarrollo Local”, Iniciativa Ciudadana, Espacio J. J. Hernández Arregui, 20 de noviembre de 2008, <https://iniciativaciudadana.wordpress. com/2008/11/20/conceptos-basicos-del-desarrollo-local/>, 5 de diciembre de 2019 .

Manzanal, Mabel; Arqueros, María Ximena; Arzeno, Mariana y Nardi, María Andrea (2009), "Desarrollo territorial en el norte argentino: una perspectiva crítica”, Revista Latinoamericana de Estudios Urbanos Regionales, 35 (105), Santiago, EURE, pp. 131-153, https://dx.doi.org/10.4067/S0250-71612009000300007

Manzanal, Mabel (2008), "Desarrollo territorial e integración nacional ¿Convergencia o divergencia?”, en José Nun y Alejandro Grimson (comps.), Territorios, identidades y federalismo, Buenos Aires, Edhasa.

Mosca, Valeria (2015), "La articulación de las políticas públicas de desarrollo rural y ordenamiento territorial: una perspectiva crítica”, Memorias de I Congreso Latinoamericano de Teoría Social, 19-21 de agosto de 2015, Buenos Aires.

Muller, Pierre (2006), Las políticas públicas, Serie estudios de caso en políticas públicas, núm. 3, Bogotá, Universidad Externado de Colombia. 
Paulsen-Bilbao, Abraham (2015), "Los aportes de Friedrich Ratzel y Halford Mackinder en la construcción de la geografía política en tiempos de continuidades y cambios", Revista de Geografía Espacios, 5 (9), Santiago de Chile, Universidad Academia de Humanismo Cristiano, pp. 64-81, <http://revistas.academia.cl/index.php/esp/ article/download/372/484/>, 8 de febrero de 2020 .

Ramírez, Blanca Rebeca (2007), "La geografía regional: tradiciones y perspectivas contemporáneas", Investigaciones Geográficas, núm. 64, Ciudad de México, UNAM, pp. 116-133, <http://www.scielo.org. $\mathrm{mx} /$ scielo.php?script=sci_arttext\&pid=S0188-461120070003 00008\&lng=es\&nrm=iso >, 3 de diciembre de 2019.

Rip, Arie (2002), "Regional innovation systems and the advent of strategic science", The Journal of Technology Transfer, 27 (1), Nueva York, Technology Transfer Society, pp. 123-131, <http://link. springer.com/article/10.1023/A:1013108906611>, 2 de diciembre de 2019 .

Santos, Milton (1993), "Los espacios de la globalización”, Anales de Geografía de la Universidad Complutense, núm. 13, Madrid, Universidad Complutense de Madrid, pp. 69-77.

Scheinkerman de Obschatko (1988), La transformación económica y tecnológica de la agricultura pampeana. 1950-1984, Buenos Aires, Ministerio de Educación y Justicia de la Nación, Secretaría de Cultura y Ediciones Culturales Argentinas.

Schejtman, Alexander y Berdegué, Julio (2004), "Desarrollo territorial rural”, Debates y Temas Rurales, Santiago de Chile, Centro Latinoamericano para el Desarrollo Rural, <https://www.rimisp.org/ wp-content/files_mf/1363093392schejtman_y_berdegue2004_ desarrollo_territorial_rural_5_rimisp_CArdumen.pdf>, $8 \mathrm{de}$ febrero de 2020.

Sol, Marie Pierre (2015), "Géographes et géographie(s) à Toulouse dans les années 1970", Bulletin de l'association de géographes français, 92 (1), Paris, Institut de Géographie, pp. 14-23, <https://journals. openedition.org/bagf/397>, 8 de febrero de 2020.

Velasco González, María (2007), "Distintos instrumentos para un mismo fin. Los instrumentos de las políticas públicas como herramienta para el análisis”, ponencia presentada en VIII Congreso Español 
de Ciencia Política y de la Administración, 18-20 de septiembre 2007, Valencia, <https://eprints.ucm.es/12184/>, 8 de febrero de 2020 .

\section{Entrevistas}

Albadalejo, Christophe (2017), “entrevista”, entrevistado por Pablo Sánchez Macchioli, Buenos Aires, 20 de febrero.

Monti, Eliseo (2017), “videoconferencia”, entrevistado por Pablo Sánchez Macchioli y Guillermo Sánchez, Buenos Aires, 6 de noviembre.

Ramilo, Diego (2017), “entrevista”, entrevistado por Pablo Sánchez Macchioli, Buenos Aires, 20 de octubre.

Anónimo 1 (2017), "entrevista”, por Pablo Sánchez Macchioli, Buenos Aires, 24 de mayo.

Anónimo 2 (2017), “entrevista”, entrevistado por Pablo Sánchez Macchioli, Buenos Aires, 20 de febrero.

Anónimo 3 (2017), "entrevista", entrevistado por Pablo Sánchez Macchioli, Buenos Aires, 22 de febrero.

Anónimo 4 (2017), “entrevista”, entrevistado por Pablo Sánchez Macchioli, Buenos Aires, 25 de mayo.

Anónimo 5 (2017), “entrevista”, entrevistado por Pablo Sánchez Macchioli, Estación Experimental Agropecuaria Área Metropolitana de Buenos Aires, INTA. Provincia de Buenos Aires, 23 de febrero.

Anónimo 8 (2017), “entrevista”, entrevistado por Pablo Sánchez Macchioli, Buenos Aires, 19 de mayo.

Anónimo 9 (2017), “entrevista”, entrevistado por Pablo Sánchez Macchioli, Buenos Aires, 10 de diciembre.

Recibido: 18 de octubre de 2019.

Reenviado: 17 de marzo de 2020.

Aceptado: 26 de marzo de 2020.

Pablo Sánchez Macchioli. Magíster en Gestión de la Ciencia, la Tecnología y la Innovación de la Universidad Nacional de General Sarmiento 
(UNGS). Actualmente, doctorando en Ciencias Sociales y Humanas por la Universidad Nacional de Quilmes (UNQ). Sus principales líneas de investigación se enfocan en el análisis de políticas científico-tecnológicas y relaciones de las instituciones de los sistemas científico-tecnológicos con sus entornos. Entre sus principales publicaciones destaca; como autor: "La gestión de la investigación y la extensión en el INTA”, Estudios Públicos, Santiago de Chile, Centros de Estudios Públicos (próximamente). Como coautor en: "Gestión y conceptualización de las interacciones con el entorno en universidades argentinas", Ciencia y Educación, Santo Domingo, Instituto Tecnológico de Santo Domingo (INTEC) (próximamente). "Instrumentos de política científica, tecnológica y de innovación en América Latina. Principales tendencias de política en Argentina, Brasil y México", en Red de Indicadores de Ciencia y Tecnología -Iberoamericana e Interamericana (RICYT), El Estado de la Ciencia 2017, Buenos Aires, RICYT, pp. 45-54 (2017). 